\title{
Eléments et fonctions du modèle de système intégré
}

\author{
Daniel Liedtke ${ }^{a}$, Ole Wiesinger ${ }^{b}$, Christian Westerhoff ${ }^{c}$, Urs Stoffeld ${ }^{\text {, }}$ Reto Stocker ${ }^{e}$, Stephan Pahls ${ }^{f}$ \\ ${ }^{a}$ Dr, COO Groupe de cliniques privées Hirslanden; ${ }^{b}$ Dr med., CEO Groupe de cliniques privées Hirslanden; ${ }^{c}$ Dr med., CCO Groupe de cliniques privées \\ Hirslanden; ${ }^{d}$ Dr med., Cabinet Chirurgical Enge, Zurich; ${ }^{e}$ Prof. Dr med., Institut d'anesthésiologie et de soins intensifs Klinik Hirslanden, Zurich; \\ fDr med., Pahls Consulting, Zürich
}

Dans la dernière édition du Bulletin des médecins suisses, nous avons présenté un modèle de système pour l'organisation du corps médical dans les hôpitaux de soins aigus [1]. Les particularités essentielles de l'élément central du modèle sont visibles sur la figure 1 . Dans cet article, nous voulons examiner de plus près une sélection d'éléments du modèle.

\section{Tâches médicales essentielles}

Une des préoccupations principales du modèle de système intégré est de permettre aux médecins hospitaliers de passer plus de temps sur leurs tâches essentielles. Parmi celles-ci, nous comptons le travail clinique auprès des patients, la formation médicale de base et continue ainsi que la recherche et l'innovation cliniques. La tâche principale des prestations pour toutes les unités de ser-

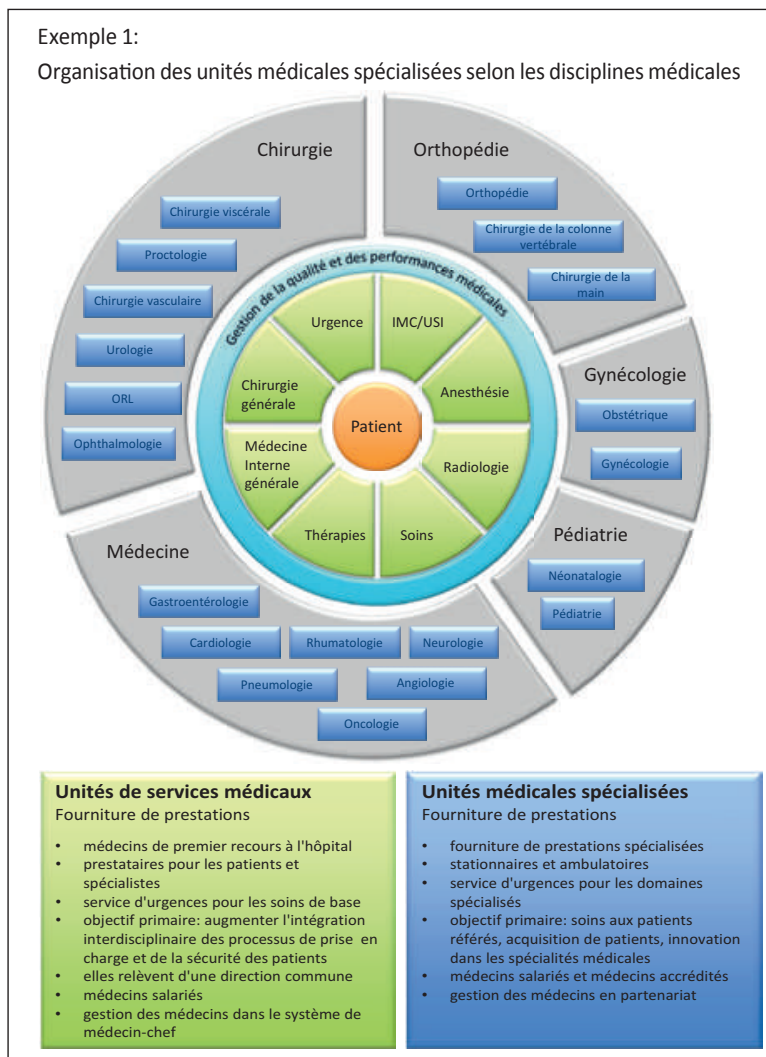

vices médicaux et unités médicales spécialisées est par conséquent d'assurer un standard élevé pour les tâches essentielles à leur domaine. Mettre un accent particulier sur les tâches essentielles a un effet positif immédiat sur les résultats et sur la satisfaction des patients, des collaborateurs et des médecins référents; cela implique également qu'il n'est plus nécessaire que chaque médecin-chef soit représenté dans la direction de l'hôpital.

\section{Les unités de services médicaux}

La responsabilité médicale de la direction dans les unités de services medicaux (ci-après: unités de services) porte avant tout, en plus des tâches intrinsèques, sur les thèmes de la qualité de service, de l'intégration des processus et de la sécurité des patients.

Exemple 2:

Organisation des unités médicales spécialisées selon les systèmes organes
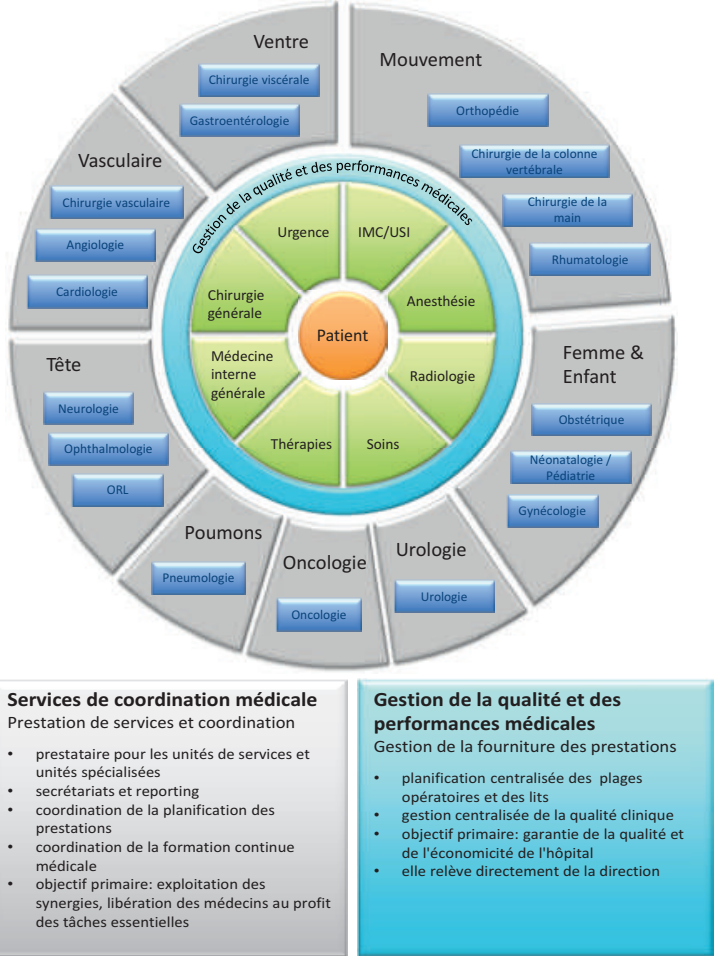

Figure 1: Possibilités d'organisation du modèle de système intégré dans les hôpitaux de soins aigus (sélection des unités spécialisées est simplement un exemple). 
En tant que médecins de premier recours en hôpital, les unités de services sont des prestataires médicaux pour les patients et les médecins des unités spécialisées. Elles gèrent l'infrastructure médicale centrale de l'hôpital (service des urgences, bloc opératoire, unité de soins intensifs, etc.) et sont, en étroite concertation avec les unités spécialisées, principalement responsables du parcours patient de l'entrée à la sortie, et donc de la sécurité des patients.

Pour obtenir une fiabilité élevée et une culture de service commune, tous les médecins des unités de services sont gérés dans le système de médecin-chef et dépendent de l'hôpital. Ces médecins-chefs, tout comme les responsables des prestations paramédicales de ces unités, dépendent, soit du directeur, soit du directeur médical de l'hôpital.

Les conflits d'intérêt éventuels entre les dirigeants des unités de services peuvent être résolus par une fonction dirigeante impartiale et centralisée, conformément aux objectifs communs de sécurité et de satisfaction des patients et d'efficience des processus. Cet accent sur les objectifs communs dans le sens d'une "culture d'entreprise» offre un barème d'indicateurs reconnu pour la résolution des conflits à l'intérieur des unités de services et vis-à-vis des unités spécialisées.

Il faut également mentionner l'importance des unités de services pour le recrutement de médecins spécialisés. Dans un contexte de compétition croissante, de hautes qualifications, la qualité de service et l'efficience des processus des soins de base intégrés à l'hôpital peuvent être décisives, pour le succès du rattachement des médecins «super-spécialisés» à l'hôpital.

\section{Les unités médicales spécialisées}

La responsabilité médicale de la direction des unités spécialisées porte avant tout, en plus des tâches intrinsèques, sur les relations avec les pourvoyeurs et l'acquisition de patients, pour lesquels la création d'une culture axée sur les patients à l'intérieur de l'unité spécialisée est indispensable.

De nombreux spécialistes réalisent une grande partie de leurs prestations en ambulatoire (pneumologie, on-
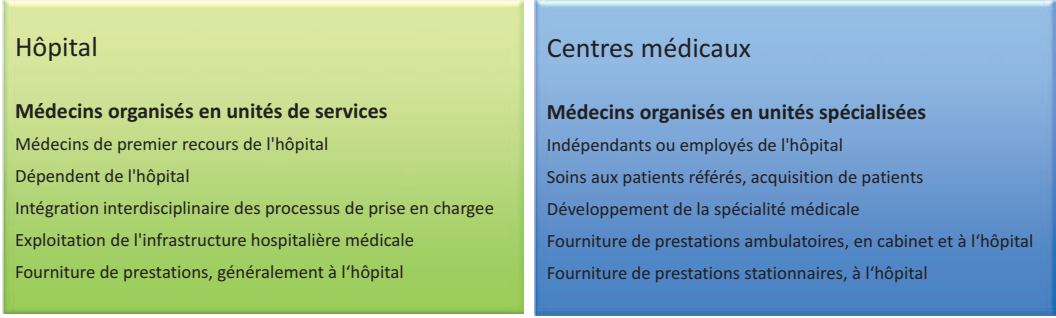

Figure 2: Exemple de répartition des unités de services médicaux et unités spécialisées médicales en hôpital et en centre médical. cologie, etc.), contribuent de manière importante à la réputation de l'hôpital et réfèrent des patients pour des traitements stationnaires. Ces médecins doivent se concentrer sur leurs tâches essentielles et peuvent, si on les dote d'une autonomie suffisante, développer encore leur spécialité médicale. Les médecins des unités spécialisés peuvent dépendre de l'hôpital ou être accrédités et doivent pouvoir s'appuyer sur une organisation collégiale (par ex. dans un système de présidence, de partenaires ou de médecin-chef).

La localisation des unités spécialisées dans un centre médical sis dans l'environnement immédiat, respectivement dans l'enceinte de l'hôpital, représente une solution de proximité intéressante, offrant des avantages tant à l'hôpital qu'aux médecins. L'activité ambulatoire au sein du centre médical peut être immédiatement combinée avec le traitement des patients stationnaires en hôpital. La proximité physique facilite l'échange interdisciplinaire entre les spécialistes et les transferts de patients et permet une intervention rapide en cas d'urgence et de complications pour les cas complexes. L'hôpital jouit d'une large disponibilité des sous-spécialités sans avoir à engager tous les spécialistes luimême; il peut plus facilement proposer aux «sur-spécialistes» de haute renommée des solutions d'emploi flexibles, selon leurs préférences personnelles (statut de salarié ou d'indépendant accrédité, à plein temps ou à temps partiel, etc.). Le centre médical regroupe les ressources spécialisées et facilite le travail à temps partiel, ce qui contribue encore à résoudre la pénurie de personnel spécialisé.

\section{Les unités de coordination médicale}

Les unités de coordination sont de petites unités de prestations de service qui coordonnent des tâches telles que la planification des piquets, le reporting, la formation médicale de base et continue, notamment pour un groupe d'unités de services et d'unités spécialisées apparentées, afin de tirer profit de synergies, générer un gain d'efficience dans les domaines non cliniques et laisser plus de place pour les tâches médicales essentielles. La direction des unités de coordination devrait en principe être régie par un système collégial, qui peut toutefois être envisagée de différentes manières. Dans les petits hôpitaux, le médecin-chef d'une unité de services peut tenir ce rôle, mais il faut alors veiller qu'il interprète cette tâche non comme le rôle classique de chef de département, mais plutôt de prestataire pour les unités de services et unités spécialisées qui lui sont attribuées. Dans les grands hôpitaux, la fonction peut être remplie par exemple par une personne qualifiée directement subordonnée à la direction. Un système 
de présidence tournante est une autre variante. Une concertation collégiale étroite avec les unités spécialisées est également décisive pour l'acceptation des unités de coordination. Au niveau des unités de coordination médicales, la formation médicale de base et continue occupe une place particulièrement importante. Une discussion approfondie sur ces tâches dépasse cependant le cadre de cette publication et devrait avoir lieu lors d'une occasion appropriée.

\section{La gestion de la qualité et des performances médicales}

La gestion de la qualité et des performances médicales occupe une place clé dans ce modèle de système intégré et devrait dépendre directement de la direction de l'hôpital. Cela doit lui garantir, dans son domaine de compétences, un pouvoir de direction efficace face aux unités de services et unités spécialisées. La direction est confiée de préférence à un spécialiste médical qualifié ayant suivi une formation complémentaire en gestion. L'acceptation et la crédibilité de la gestion de la qualité et des performances dépend de la nomination de personnes effectuant leurs tâches de manière ouverte et transparente, en concertation avec les unités de service et les unités spécialisées.

\section{Gestion centralisée des performances}

Une prémisse importante du modèle de système intégré est l'abandon de la notion de «départements gérés par lit» et de la souveraineté du chirurgien sur la planification des opérations. A l'heure des forfaits par cas, un taux élevé d'occupation et d'opération est décisif pour la réussite économique d'un hôpital et ne peut être atteint que par une planification centralisée et flexible. Il est difficile de l'atteindre quand les lits ou les salles d'opérations sont affectés à des départements déterminés et quand les médecins-chefs en gèrent l'occupation. Dans ce modèle de système intégré, les capacités des unités de services sont mises à disposition, des spécialistes travaillant dans l'hôpital et de leurs patients, suivant la demande. Cela concerne à parts égales les salles d'opération, les salles d'intervention, l'unité de soins intensifs et les unités de lits. Le service de gestion des plages opératoires et des lits assure, en concertation continue avec toutes les unités, que les patients soient suivis dans l'infrastructure appropriée, bénéficient des compétences spécialisées requises, le tout en veillant à ce qu'un niveau d'occupation élevé soit maintenu.

En définissant de cette manière la responsabilité des spécialistes non plus par la ressource «lit» ou "salle d'opération" mais par la focalisation sur le cas traité, les patients peuvent, dans une salle d'opération et dans une unité de lits déterminés, être suivis médicalement par plusieurs unités spécialisées, sans conflit de compétences, ce qui facilite entre autres l'intégration des médecins accrédités. Ce concept de «direction par le cas plutôt que par le lit» implique un changement culturel fondamental, qui exige pour de nombreux médecinschefs hospitaliers la renonciation à des privilèges historiques.

\section{Gestion centralisée de la qualité clinique}

Presque tous les hôpitaux suisses ont institutionnalisé la gestion de la qualité au niveau de l'entreprise et emploient des coordinateurs qualité qualifiés, ce qui retire beaucoup de travail aux cliniciens, par exemple pour la documentation de leurs processus, la mise en œuvre des systèmes de qualité (par ex. CIRS) ou encore pour ce qui concerne l'hygiène hospitalière et les mesures de la qualité. De nombreuses tâches importantes de

\section{Ce concept de «direction par le cas plutôt}

que par le lit» implique un changement culturel fondamental.

gestion de la qualité médico-clinique (standards de prise en charge, colloques pluridisciplinaires d'indications, conférences sur la morbidité et la mortalité, direction des registres, etc.) restent toutefois aussi fragmentées que les structures de gestion médicales. Lorsque chaque département médical gère ses propres normes et commissions, la concertation interdisciplinaire est complexe. Il règne un peu partout une divergence frappante entre la gestion commune de la qualité centralisée, en général bien dotée en personnel/professionnels, et une variété de solutions décentralisées de gestion de la qualité clinique, souvent mal dotées en personnel. En charger à temps partiel les spécialistes arrivés en dernier, qui devraient travailler principalement sur les patients, ou mobiliser à cet effet des secrétariats ou médecins-assistants insuffisamment qualifiés, est toutefois de moins en moins acceptable.

Centraliser également la gestion de la qualité clinique et l'attribution de spécialistes qualifiés serait un pas important vers plus de professionnalisme et d'efficience. La communication des résultats serait plus objective et transparente et la probabilité que les mesures d'amélioration de la qualité soient mises en place de façon cohérente plus élevée.

La détermination des normes de traitement et de qualité spécifiques aux spécialités médicales reste par ailleurs, de manière indiscutable, de la responsabilité de la direction des unités de services respectivement des unités spécialisées médicales. Aucune unité exogène ne peut leur retirer cette tâche spécialisée. La gestion centra- 
lisée de la qualité clinique peut toutefois exiger que les normes soient déterminées de manière interdisciplinaire, que la totalité du parcours médical soit assurée conformément aux bonnes pratiques et aux critères EAE (efficacité, adéquation, économicité). Tandis que les médecins gardent la souveraineté spécialisée sur les normes de traitement et de qualité cliniques, la gestion centralisée de la qualité améliorera principalement la gestion et renforcera la fiabilité.

\section{Tâches de la direction de l'hôpital}

Comme notre modèle pose la description des tâches et principes de direction au premier plan, il n'est pas lié à une structure organisationnelle déterminée. La mise en œuvre du modèle peut fonctionner avec des organigrammes différents. Pour cette raison, le modèle ne prescrit par exemple pas quels services, professions ou fonctions doivent être représentés dans la direction de l'hôpital. C'est à chaque hôpital d'en décider individuellement. On peut toutefois déduire du modèle quelques recommandations pour les niveaux de direction supérieurs de l'hôpital:

- La direction opérationnelle de toutes les unités de services devrait être hiérarchique et, idéalement, regroupée sous un dirigeant commun qui, en raison de sa grande importance, devrait être membre de la direction de l'hôpital.

- La direction opérationnelle de chaque unité spécialisée et unité de coordination médicale peut être basée sur un management par objectifs financiers et de prestations, mais elle ne devrait pas être gérée par la direction des unités de services, afin d'assurer une séparation effective des pouvoirs.

- La direction opérationnelle de la gestion de la qualité et des performances médicales est prise en charge par un responsable, de préférence directement subordonné à la direction de l'hôpital. Il ne devrait pas relever de la direction de l'unité de services, afin d'assurer une séparation effective des pouvoirs. Cette personne doit toutefois entretenir des relations ouvertes et transparentes avec toutes les unités médicales, afin de garantir une large acceptation.

- Le développement stratégique des unités de services et unités spécialisées médicales (personnel, processus, technologie, infrastructure) est à mettre sur le même pied que le développement du portefeuille de prestations de l'ensemble de l'hôpital et devrait pour cette raison être géré par la direction de l'hôpital. La concertation étroite avec les unités de services et les unités spécialisées ainsi que la délégation de projets de développement à ces dernières devraient toutefois être une évidence.

\section{Introduction du modèle}

Le modèle de système intégré décrit ici ne se présente pas comme un projet clé en mains pour la réorganisation des hôpitaux. Par ce modèle, nous souhaitons plutôt montrer les éléments et principes qui ont fait leurs preuves à la Clinique Hirslanden. Nous sommes convaincus que les éléments décrits peuvent être généralisés et que ce modèle d'hôpital peut aider à améliorer les performances de votre système de gestion, indépendamment de sa forme juridique ou de son propriétaire. Pour le mettre en place, il est indispensable que la direction de l'hôpital et le corps médical se défassent de structures et représentations des rôles dépassées, qu'ils définissent clairement les tâches médicales et qu'ils les attribuent en répartissant le travail par unités ad hoc. Chacun doit avant tout faire ce qu'il sait faire le mieux. Ce modèle de système intégré ne donne aucune prescription pour la désignation d'unités fonctionnelles et de fonctions de direction. Des notions éprouvées telles que «département», "chef de service», "médecin-chef» ou «médecin adjoint» peuvent toujours être utilisées, mais il faudrait vérifier leur signification et les adapter au nouveau projet. L'organisation et la direction de chaque unité fonctionnelle doivent être subordonnées et adaptées sans "esprit de clocher» aux tâches spécifiques dans le cadre du processus de gestion des patients. Les compétences pour les tâches-clés médicales devraient d'ailleurs toujours être déléguées au niveau hiérarchique le plus proche possible des patients, tandis que les tâches de coordination et d'administration sont à centraliser autant que possible, afin de donner aux spécialistes hautement qualifiés plus de place pour leurs tâches essentielles.

\footnotetext{
Références

1 Liedtke D, Wiesinger O, Westerhoff C, Stoffel U, Stocker R, Pahls S. Modèle d'un système intégré innovant pour les hôpitaux de soins aigus. Bull Méd Suisses. 2015;96(44):1625-8.

- Littérature disponible chez Daniel Liedtke.
}

Remerciement

Nous remercions Cédric Bossart pour la traduction de cet article. 